\title{
COMENTARIO EDITORIAL Incertidumbre y probabilidad en medicina
}

Para comenzar este editorial presentaremos una situación utópica en la cuál el hallazgo clínico de una enfermedad o condición se presente de la siguiente manera: 1 ) esta siempre presente en los pacientes con la enfermedad (si el hallazgo está ausente, la enfermedad está ausente); 2) nunca esta presente en los pacientes que no tienen la enfermedad (en este caso, si el hallazgo está presente, la enfermedad está presente).

Todos sabemos que en la práctica de la medicina este tipo de situaciones ocurren en forma excepcional (ej. el anillo de Kayser y Fleisher sólo aparece en los pacientes con enfermedad de Wilson, mientras que es muy raro que un paciente con insuficiencia cardíaca descompensada no tenga taquicardia).

El médico se ve obligado a trabajar en un estado de incertidumbre ya que generalmente sólo cuenta con información incompleta para inferir "lo que le pasa al paciente". Para colmo, a veces estas pistas no reflejan el verdadero estado del paciente (ej. los datos del interrogatorio, examen físico y análisis complementarios pueden dar resultados que conduzcan a conclusiones erróneas).

Ejemplo: el cansancio es un síntoma común de depresión y tambien de anemia; mientras que una pérdida de peso inexplicada puede ser consecutiva a hipertiroidismo, cáncer, SIDA, trastorno de la alimentación, hipoalimentación por pobreza, etc.

\section{La probabilidad como un código para expresar incertidumbre}

Cuando los médicos decimos que una entidad clínica se ha presentado con un patrón clásico o de "libro"; cuando expresamos frente a un paciente con cierta sintomatología que una enfermedad "posiblemente" está presente o que alguna otra "probablemente" no lo está; o bien, al referirnos a una condición la rotulamos de "rara" o "frecuente", no hacemos otra cosa que asignar nuestras propias y personales probabilidades, que surgen de nuestra experiencia clínica, de lo que hemos leído y de comentarios de otros colegas.

¿Pero qué queremos decir cuando mencionamos que algo es raro o frecuente? Por un lado las personas pueden eligir distintas palabras para expresar lo mismo acerca de la probabilidad de un evento; o bien por otro, escoger el mismo vocablo para referirse a probabilidades muy distintas.

Ejemplo: algunos estudios realizados en individuos no hispanoparlantes mostraron que cuando alguien dice "altamente probable" puede estar pensando en un rango que va desde un 60 a un $99 \%$; mientras que si la palabra es "habitualmente", el rango oscila entre 15 y $99 \%$ y si la frase es "no puede ser excluido" este va de 7 a $98 \%$, etc.) ${ }^{1}$

Por lo tanto, la interpretación de estas probabilidades puede resultar confusa o ambigua.

Ejemplo: un médico puede decir que una enfermedad es frecuente porque vió diez pacientes en su vida, otro puede llamar frecuente sólo a las enfermedades que se le presentan varias veces por mes y un tercero, sólo a las que ve por lo menos semanalmente.

El uso de probabilidades en lugar de palabras permite expresar cuantitativamente la incertidumbre para que cualquier dato clínico adicional ajuste la hipótesis diagnóstica sobre bases más racionales. Podemos, entonces, definir la probabilidad como un número entre 0 y 1 que encierra una opinión sobre la posibilidad que un evento ocurra.

\section{Llevando la probabilidad a la práctica}

Cuando un médico ve a un paciente y le solicita un estudio diagnóstico, debe pensar para qué lo pide y de qué manera el resultado cambiará el diagnóstico o la conducta a seguir. El impacto que la nueva información produce en la modificación del juicio clínico depende de la probabilidad asignada antes de conocerla. En otras palabras, de lo que se ha dado en llamar probabilidad previa o probabilidad pre-prueba (en inglés: pre-test).

La elección dependerá de la probabilidad que el médico le adjudica a su hipótesis. Si ésta es muy baja, lo más sensato es no hacer nada y si ésta es muy alta, considerar directamente la administración de un tratamiento. Por lo tanto, un concepto importante que queremos transmitir es que la situación en que las pruebas diagnósticas tienen el más alto impacto es dentro del rango de probabilidades previas intermedias ${ }^{2}$. Ver figura 1.

Figura 1

Probabilidad y Modelo Umbral

\begin{tabular}{|l|c|}
\hline $\begin{array}{l}\text { No Prueba } \\
\text { No tratamiento }\end{array}$ & Prueba \\
\hline $0-$ probabilidad & Tratamiento \\
\hline $\begin{array}{l}\text { Certeza de } \\
\text { No- enfermedad }\end{array}$ & $\begin{array}{r}\text { Certeza de } \\
\text { Enfermedad }\end{array}$ \\
Cuando la probabilidad es baja la mejor opción es no realizar estu- \\
dios diagnósticos ni efectuar tratamiento, cuando la probabilidad es \\
intermedia corresponde pedir un test diagnóstico y cuando la proba- \\
bilidad es alta se debería tratar al paciente ya que los estudios diag- \\
nósticos no aportan mayor información. En este último caso los es- \\
tudios pasan a ser pronósticos.
\end{tabular}

Ejemplo 1: un hombre de 55 años hipertenso consulta por una historia de cuatro semanas de dolor precordial opresivo vinculado al esfuerzo que cede luego de dos o tres minutos de reposo. El médico considera firmemente el diagnóstico de angina de pecho y le pide un electrocardiograma de esfuerzo, que es normal. Ejemplo 2: una mujer de 35 años sin factores de riesgo coronarios, consulta por síntomas de ardor retroesternal no relacionados con esfuerzos. A pesar de que no parece una isquemia miocárdica, el médico decide pedirle un prueba ergométrica de esfuerzo.

Ejemplo 3: un hombre de 45 años fumador, consulta por tres semanas de dolor precordial, a veces opresivo y a veces punzante, inconsistentemente relacionado con el esfuerzo. El médico cree que puede tratarse de una angina de pecho de presentación atípica y se pregunta si un electrocardiograma de esfuerzo podría ayudar a corroborar éste diagnóstico.

De acuerdo al estudio de Diamond y Forrester ${ }^{3}$, Las probabilidades previas de enfermedad coronaria correspondientes a estas presentaciones serían: $90 \%$ en el ejemplo 1, $5 \%$ en el 2 y $50 \%$ en el 3 . En el ejemplo 1, si la prueba es positiva, la probabilidad posterior de que se trate de una cardiopatía isquémica (probabilidad que resulta luego de efectuar la prueba) aumenta al. $98 \%$, lo que a la hora de tomar decisiones de manejo no cambia mucho las cosas, ya que ésta antes era del $90 \%$. Por otro lado, si la ergometría es negativa la probabilidad de enfermedad coronaria disminuye a $80 \%$. Sin embargo, a pesar de éste resultado, la presencia de angina de pecho es todavía cuatro veces más probable que cualquier otro diagnóstico alternativo, lo que seguramente preservará en el pensamiento del médico la impresión de que "el paciente probablemente tenga enfermedad coronaria".

En el ejemplo 2, si la prueba es negativa, la probabilidad disminuye a $2 \%$, lo que tampoco cambia mucho las cosas (antes era de $5 \%$ ). Pero si es positivo sólo la aumenta a $26 \%$. A pesar de este resultado, la presencia de otra causa que explique los síntomas del pacien- 
te es tres veces más probable que la angina de pecho, lo que seguramente obligará al médico tratante a realizar otra prueba que dirima la incertidumbre.

En el ejemplo 3, si la prueba es positiva, la probabilidad aumenta a $87 \%$ y si es negativa, disminuye a $31 \%$. En ambas situaciones, la ergometría permite diferenciar claramente cursos alternativos de acción tales como iniciar un tratamiento específico o bien, esperar y observar. Podemos decir que, desde un punto de vista estrictamente diagnóstico, en el primer y segundo ejemplo la prueba fue inútil ya que no la información adicional que brindó no fue suficiente para decidir una conducta distinta a la que el médico, probablemente, adscribiera antes de solicitar la prueba: tratar al paciente con aspirina, bboqueantes, etc. en el primer caso; y no tratarlo en el segundo. De este modo, independientemente de la prueba que usemos, la probabilidad de enfermedad a la que se arriba luego de conocerse el resultado de la prueba depende en gran medida de cual era la probabilidad previa a su realización (cuanto mayor es la primera, mayor la segunda y viceversa) ${ }^{4}$.

La definición de probabilidad toma en cuenta toda la información disponible para el médico al momento de efectuar su juicio clínico. Esta puede categorizarse en información proveniente de la experiencia personal e información proveniente de la experiencia publicada por otros. Estas deben ser combinadas para establecer correctamente un anclaje * en la estimación de la probabilidad previa.

\section{Experiencia personal}

Sin duda, cuando estiman probabilidades, los médicos utilizan su experiencia previa con casos que comparten características con el actual. Los clínicos experimentados han visto tantos pacientes que poseen una innata capacidad intuitiva para diferenciar eventos comunes de aquellos que no lo son. Concluimos que la experiencia personal del médico es el factor más influyente en la determinación de las probabilidades "pre test". Sin embargo, como cualquier otro análisis subjetivo y teniendo en cuenta que este proceso depende de inferencias a partir de datos "guardados" en la memoria, no está exento de errores de apreciación y sesgos que pueden, en algunos casos, distorsionar su interpretación.

\section{Experiencia publicada}

La publicación de experiencias en las revistas científicas o en los libros de texto es una invalorable fuente de información cuando se trata de estimar probabilidades. Podemos decir que los estudios publicados son útiles por varias razones: a) un trabajo sobre una enfermedad rara habitualmente refleja una experiencia que ni el más experimentado de los médicos podría adquirir por si sólo; b) los análisis estadísticos permiten organizar la información en una forma particularmente útil para facilitar la toma de decisión; c) los estudios publicados permiten registrar las prevalencias de enfermedades para determinados hallazgos en una población, así como la prevalencia de hallazgos para una enfermedad dada.

Estas estimaciones son frecuentemente utilizadas como probabilidades de referencia para determinar la probabilidad de comienzo (ancla) y ajustar luego, teniendo en cuenta la experiencia personal y las características del paciente. Sin embargo es importante tener en mente un importante sesgo en éste tipo de estimaciones: el que sobreviene cuando se extrapolan directamente las prevalencias obtenidas en el trabajo a las de la población de la cual proviene nuestro paciente. El problema radica en que, en general, las publicaciones provienen de grandes centros que manejan poblaciones muy seleccionadas (ej. pacientes derivados a especialistas que raramente ven sujetos con hallazgos clínicos que implican probabilidades bajas de enfermedad). La prevalencia de condiciones serias dado un hallazgo determinado es mucho más alta que la que ocurre, por ejemplo, en un centro de atención primaria o en un consultorio general. Dado que ésta distribución de frecuencias está notoriamente afectada por los filtros de referencia (derivación) sólo es válida para los centros que manejan poblaciones similares. Los médicos generales deben tener especial cuidado cuando aplican a su práctica habitual las prevalencias de las publicaciones que han leído.

Ejemplo: una mujer de 35 años concurre a un consultorio general por varios registros de presión arterial de 150/100. Los análisis iniciales son normales. El médico, luego de haber leído un trabajo proveniente de un centro terciario que reportaba una prevalencia de $7 \%$ de hipertensión secundaria de origen renovascular, solicita un radiorenograma isotópico y una angiografía renal para descartar esta entidad.

Un trabajo proveniente de un centro de atención primaria reportó para la misma enfermedad, una prevalencia de $0.2 \%$. Si el médico hubiera contado con éste dato no se habría apresurado a estudiar tan rápidamente a la paciente. Cada tipo de médico lee (o debería leer) publicaciones referidas a pacientes tratados por su grupo de pertenencia. Esta realidad debe ser contemplada cuando no existe acuerdo entre distintos médicos sobre las probabilidades asignadas a la condición del paciente.

Como vemos, este tema no se agota fácilmente. En números posteriores de Evidencia continuaremos desarrollando otros aspectos del uso de la probabilidad en medicina, en la toma de decisiones y en el manejo de la información al paciente; así como algunos tópicos en los que existe controversia al respecto como los referidos a la extrapolación de probabilidades al paciente individual o riesgos que surgen de estudios poblacionales 5 .

Dr. Adolfo Rubintein - Dr. Alberto Velázquez

Unidad de Medicina Familiar y Preventiva. Hospital Italiano de Buenos Aires.

\footnotetext{
Referencias

1. Norman G. y Streiner D.. Health measurements scales. Oxford University Press, 1995Cap. 4: Scaling responses.Página 37.

2. Department of Clinical Epidemiology and Biostatistics, Mc Master University Health Sciences Centre. How to read clinical journals: To learn about a diagnostic test. Can. Med.As-

soc.J. 1981;124:703-10

3. Diamond G, Forrester J. Analysis of probability as an aid in the clinical diagnosis of coronary artery disease. N EngL J Med. 1979;300:1350-1357

4. Sox H Jr, Blatt M, et. al. Medical Decision Making. Butterworth Publishers, 1988.

5. Goodman S. Probability at the Bedside: The Knowing of Chances or the Chances of Knowing?Annals of InternaL Medicine, 6 April 1999. 130:604-606.
} 